\title{
Cavernous sinus thrombophlebitis causing reversible narrowing of internal carotid artery
}

\author{
K. S. M adhusudhan, H arsh Kandpal, Jitesh Ahuja \\ Department of Radiodiagnosis, All India Institute of Medical Sciences, New Delhi-110 029, India
}

A nine-year-old boy presented with eight-days history of progressive frontal headache, high-grade fever, periorbital swelling and proptosis. Clinical examination showed tenderness in the region of frontal sinus, chemosis and restricted right ocular movements. Contrast-enhanced magnetic resonance imaging (MRI) performed with the clinical suspicion of cavernous sinus thrombophlebitis (CST) showed mucosal enhancement of all the paranasal sinuses and frontal pachymeningitis [Figure 1a]. The right cavernous sinus was enlarged and also showed contrast enhancement [Figure 1b]. The wall of the petrous segment of the right internal carotid artery (ICA) showed diffuse enhancement [Figure 2a]. 3D contrast-enhanced MR angiogram (MRA) revealed diffuse smooth narrowing of the cavernous and petrous segments of internal carotid artery (ICA) [Figure 2b]. Based on the imaging features, a diagnosis of pansinusitis causing right CST, arteritis and narrowing of intra-cavernous ICA, and frontal pachymeningitis was made. The patient was started on broad-spectrum antibiotics. Follow-up MRI performed

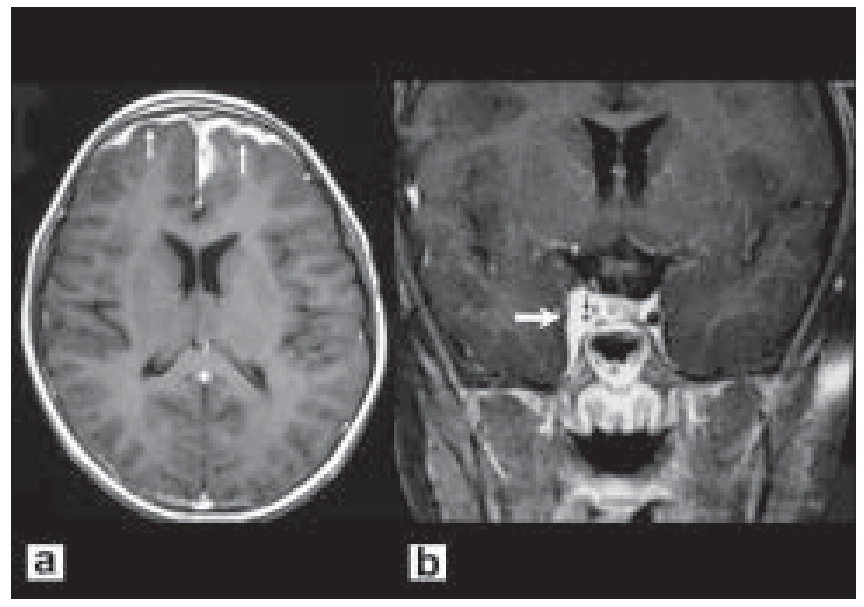

Figure 1: Axial contrast-enhanced T1W MRI (a) shows thick enhancement of the frontal duramater (arrows). Coronal T1W contrastenhanced MRI (b) shows diffuse enhancement of the cavernous sinus (white arrow) with narrowing of the ICA (small arrow) two months later showed resolution of sinusitis, CST and restoration of ICA caliber to normal [Figure 3].

Cavernous sinus thrombophlebitis is a rare, potentially lethal condition which still has a mortality of about $30 \%{ }^{[1]}$ The cavernous sinus has extensive valveless communications with many facial and neck veins which can serve as conduits for spread of infection. Infection typically spreads from the adjacent paranasal sinuses, particularly sphenoid and ethmoid sinuses, face, orbit or teeth. Staphylococcus aureus is the most common causative organism. ${ }^{[1]}$ Infection of the cavernous sinus may present as fever, ptosis, proptosis, chemosis, cranial nerve palsies, headache and periorbital edema. ${ }^{[1]}$ Neurological sequelae may occur uncommonly in the form of ophthalmoplegia, blindness, hemiparesis and pituitary insufficiency. ${ }^{[2]}$ Involvement of the ICA is uncommon and occurs in the form of stenosis, occlusion and aneurysm formation. ${ }^{[2,3]}$ Although the location of the ICA within the cavernous sinus may indicate that its involvement is likely in cases of CST, in practice, it

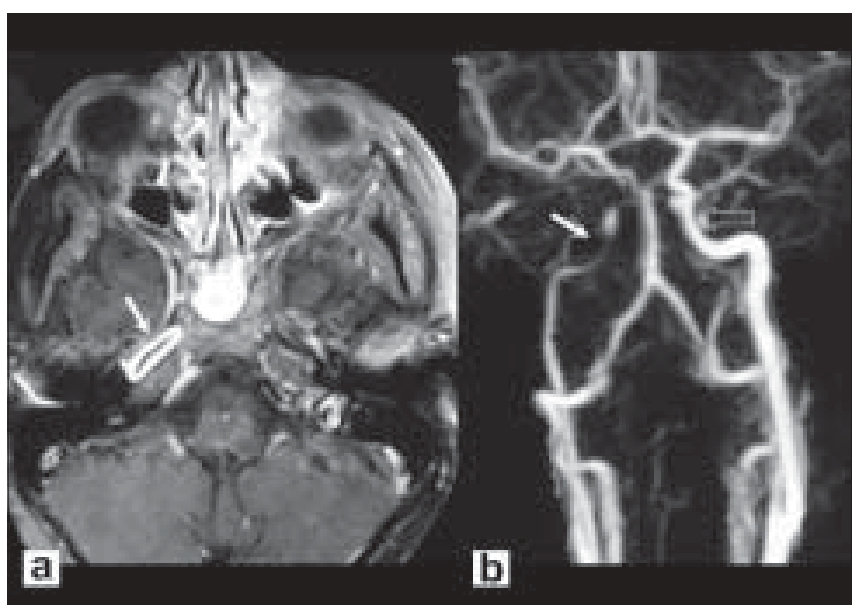

Figure 2: Axial contrast-enhanced T1W MRI (a) shows diffuse enhancement of the wall of the right intra-petrous ICA (arrow). Ethmoid and sphenoid sinusitis is also seen. 3D contrast-enhanced MR angiogram (b) shows long segment narrowing of the right ICA (arrow). The left ICA is normal (black arrow) 


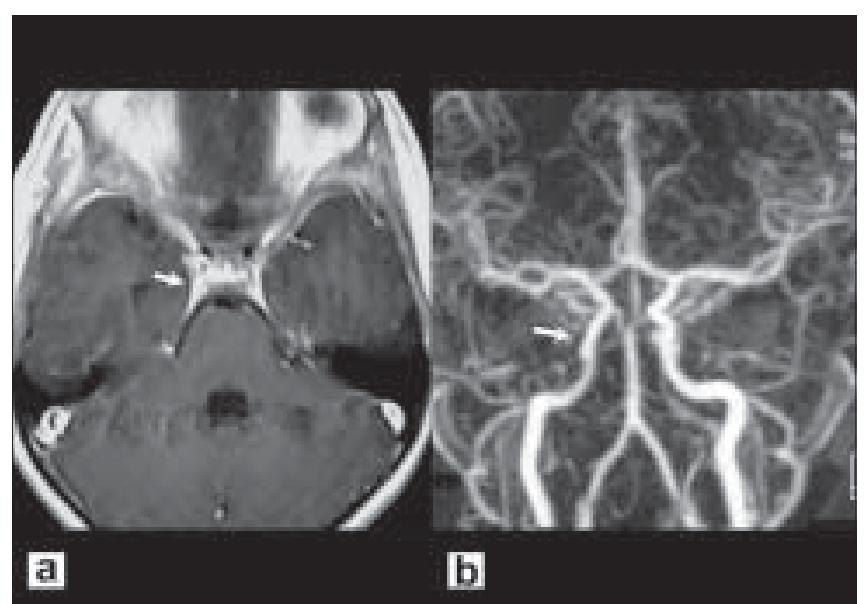

Figure 3: MRI done after two months of antibiotic therapy. Axial T1W contrast-enhanced MRI (a) shows normal size and enhancement of the right cavernous sinus (arrow). The MR angiogram (b) shows reversal of right ICA narrowing (arrow)

is seldom seen. The inflammation involves the arterial wall, leading to stenosis, luminal thrombosis and aneurysm formation due to the weakening of the wall. Stenosis may also develop secondary to compression of the artery by the adjacent thrombosis. ${ }^{[1]}$

The major role of imaging is in detection of the cause and extent of inflammation. Computed tomography (CT) scan and more importantly, MRI has contributed significantly towards diagnosis. ${ }^{[1]}$ Direct signs of CST include enlargement of the sinus with a lateral bulge, and multiple irregular filling defects. ${ }^{[4,5]}$ Indirect signs include enlargement of the tributary veins, particularly superior ophthalmic vein, exophthalmos, soft tissue edema and abnormal dural enhancement of the lateral wall of the sinus. ${ }^{[5]}$ In addition, the source of infection in the paranasal sinuses, orbit or mastoid can be demonstrated. MR angiography is essential for the evaluation of ICA.

The mainstay of treatment in CST is systemic administration of broad-spectrum antibiotics. ${ }^{[1]}$ Therapy has to be continued for about three to four weeks after the signs of infection have ceased. Surgery is needed for the management of primary cause or complications.

\section{Address for correspondence: Dr. Harsh Kandpal, Senior Research Associate, All India Institute of Medical Sciences, New Delhi - 110 029, India. E-mail: drharshk@yahoo.com}

DOI: $10.4103 / 0028-3886.48792$

\section{References}

1. Ebright JR, Pace MR, Niazi AF. Septic thrombosis of the cavernous sinuses. Arch Intern Med 2001;161:2671-6.

2. Hoshino C, Satoh N, Sugawara S, Kuriyama C, Kikuchi A, Ohta M. Septic cavernous sinus thrombosis complicated by narrowing of the internal carotid artery, subarachnoid abscess and multiple pulmonary septic emboli. Intern Med 2007;46:317-23.

3. Ozuer MZ, Ardic FN, Coskun E, Yalcin AN, Akkoyunlu NS. Carotid artery occlusion due to bacterial paranasal sinusitis. Ann Otol Rhinol Laryngol 1999;108:87-90.

4. Ahmadi J, Keane JR, Segall HD, Zee CH. CT observations pertinent to septic cavernous sinus thrombosis. AJNR Am J Neuroradiol $1985 ; 56: 755-8$.

5. Schuknecht B, Simmen D, Yuksel C, Valavanis A. Tributary venous sinus occlusion and septic cavernous sinus thrombosis: CT and MR findings. AJNR Am J Neuroradiol 1998;19:617-26.

Accepted on 26-01-2009

Source of Support: Nil, Conflict of Interest: None declared. 